INTERVENTIONAL CARDIOLOGY AND SURGERY

\title{
Relation between coronary pressure derived collateral flow, myocardial perfusion grade, and outcome in left ventricular function after rescue percutaneous coronary intervention
}

\author{
K P Balachandran, C Berry, J Norrie, B D Vallance, M Malekianpour, T J Gilbert, A C H Pell, \\ K G Oldroyd
}

Heart 2004;90:1450-1454. doi: 10.1136/hrt.2003.023606

\begin{abstract}
See end of article for authors' affiliations

Correspondence to: Dr Kanarath P

Balachandran, Camden

House, Bristol Royal

Infirmary, Bristol,

BS2 8HW, UK;

kanarath_balachandran@

hotmail.com
\end{abstract}

Accepted 1 March 2004

\begin{abstract}
Objective: To evaluate the relation between pressure derived coronary collateral flow (PDCF) index and angiographic TIMI (thrombolysis in myocardial infarction) myocardial perfusion (TMP) grade, angiographic collateral grade, and subsequent recovery of left ventricular function after rescue percutaneous coronary intervention (PCI) for failed reperfusion in acute myocardial infarction.

Methods: The pressure wire was used as the guidewire in 38 consecutive patients who underwent rescue PCl between December 2000 and March 2002. Follow up angiography was performed at six months. Baseline and follow up single plane ventriculograms were analysed off line by an automated edge detection technique. A linear model was fitted to assess the relation between 0.1 unit increase in PDCF and change in left ventricular regional wall motion.

Results: Patients with TMP 0 grade had significantly higher mean (SD) PDCF than patients with TMP 1-3 $(0.30(0.11) \vee 0.15(0.07), p<0.0001, r=-0.5)$. A similar relation was observed between TMP grade and coronary wedge pressure (mean (SD) 28 (16) $\mathrm{mm} \mathrm{Hg}$ with TMP 0 v 9 (7) $\mathrm{mm} \mathrm{Hg}$ with TMP 1-3, $p=0.001, r=-0.4)$. Higher PDCF was associated with increased left ventricular end diastolic pressures $(0.28(0.14)$ with end diastolic pressure $>20 \mathrm{~mm} \mathrm{Hg} v 0.22(0.09)$ with end diastolic pressure $<20 \mathrm{~mm} \mathrm{Hg}, \mathrm{p}=0.08, r=0.2$ ). No correlation was observed between PDCF and Rentrops collateral grade $(0.26(0.13)$ with grade $0 \vee 0.25(0.11)$ with grades $1-3, p=0.4, r=-0.06)$. No linear relation existed between changes in PDCF and changes in left ventricular regional wall motion.

Conclusion: PDCF in the setting of rescue PCI for failed reperfusion after thrombolysis does not predict improvement in left ventricular function. Increased PDCF and coronary wedge pressure in acute myocardial infarction reflect a dysfunctional microcirculation rather than good collateral protection.
\end{abstract}

$\mathrm{D}$ istal intracoronary pressure $(\mathrm{Pd})$ and myocardial fractional flow reserve (FFRmyo) can be measured with the pressure wire. Resistance is abolished under conditions of maximum hyperaemia, thereby establishing a direct relation between pressure and flow. In the setting of chronic stable angina, FFRmyo of $\leqslant 0.75$ suggests a functionally significant lesion. Mean Pd during balloon occlusion is the coronary wedge pressure $(\mathrm{Pw})$ and this is dependent on collateral circulation. The pressure derived coronary collateral flow (PDCF) index can be obtained from the mean $\mathrm{Pw}$, mean aortic pressure $(\mathrm{Pa})$, and mean central venous pressure $(\mathrm{Pv})($ where $\mathrm{PDCF}=\mathrm{Pw}-\mathrm{Pv} / \mathrm{Pa}-\mathrm{Pv}) \cdot{ }^{1-5}$ In patients with chronic stable angina higher PDCF has been shown to reflect collateral protection and is associated with improved outcomes after percutaneous coronary intervention (PCI). ${ }^{6}$ The significance of increased Pw during PCI for acute myocardial infarction is uncertain. One group has reported a good correlation with collateral flow but two more recent studies did not confirm this and suggested instead that increased PW reflects microvascular dysfunction. ${ }^{7-9}$ In this study we have measured PDCF in the setting of rescue PCI after failed thrombolysis in acute myocardial infarction, assessing its ability to predict outcomes in left ventricular function and exploring correlations with angiographic TIMI (thrombolysis in myocardial infarction) myocardial perfusion (TMP) grade, which is a marker of microvascular dysfunction after reperfusion, left ventricular end diastolic pressure (LVEDP), and Rentrops angiographic collateral grade.

\section{METHODS}

Thirty eight consecutive patients who underwent rescue PCI for failed reperfusion between December 2000 and March 2002 consented to take part in this study. Failed reperfusion after thrombolysis was diagnosed on the basis of standard ECG criterion: failure of the peak ST segment elevation to resolve by more than $50 \%$ in the lead with maximum ST elevation at 90 minutes after initiation of thrombolytic treatment. Written informed consent was obtained and after local anaesthesia with $1 \%$ lidocaine, PCI was performed through the right femoral artery with 6 French guide catheters (Cordis, Miami, Florida, USA) without side holes. Heparin was administered as a bolus intra-arterially at a dose of 50-100 U/kg, and intracoronary glyceryl trinitrate ( $100 \mu \mathrm{g}$ ) was administered after selective engagement of the infarct related artery.

\section{Coronary pressure recordings}

The 0.014 inch pressure wire 3 (Radi Medical Systems, Uppsala, Sweden) was used as the primary guidewire through the 6 French guiding catheter. The wire was set at

Abbreviations: FFRcor, coronary fractional flow reserve; FFRmyo, myocardial fractional flow reserve; LVEDP, left ventricular end diastolic pressure; $\mathrm{Pa}$, aortic pressure; $\mathrm{PCl}$, percutaneous coronary intervention; $\mathrm{Pd}$, distal intracoronary pressure; $\mathrm{PDCF}$, pressure derived coronary collateral flow; Pv, central venous pressure; Pw, coronary wedge pressure; TIMI, thrombolysis in myocardial infarction; TMP, TIMI (thrombolysis in myocardial infarction) myocardial perfusion 
0, calibrated, and manipulated beyond the occluded or stenosed segment. Despite the pressure wire being generally less flexible than standard guidewires, in only two patients was additional backup support of a balloon necessary to cross the occluded segment. Intracoronary boluses of adenosine were used to achieve maximum hyperaemia: $40 \mu \mathrm{g}$ in the left coronary artery and $20 \mu \mathrm{g}$ in the right coronary artery. Pd was obtained from the pressure wire and Pa was monitored at the tip of the guiding catheter with a fluid filled system. FFRmyo was calculated as the ratio of mean Pd to mean Pa under conditions of maximum hyperaemia (where FFRmyo $=\mathrm{Pd} /$ $\mathrm{Pa})$. PDCF was calculated from the ratio of mean $\mathrm{Pw}$ (Pd during balloon inflation and complete coronary occlusion) and mean $\mathrm{Pa}(\mathrm{PDCF}=\mathrm{Pw} / \mathrm{Pa})$. In all patients the $\mathrm{PW}$ obtained during the first balloon inflation was used to calculate PDCF. The balloon was not inflated sequentially to enable multiple recordings of Pw, as we felt that this would compromise clinical judgement and patient care. LVEDP was recorded and single plane left ventriculography was performed at the end of the procedure. Mean Pv was not routinely estimated. The use of stents and glycoprotein IIb/ IIIa blockers was at the discretion of the attending cardiologist. Follow up cardiac catheterisation including left ventriculography was performed at six months in 33 patients (one patient died during follow up and four patients declined to undertake the six month repeat angiogram after having consented to the study).

Primary exclusion criteria were cardiogenic shock, failure to obtain consent, and significant co-morbidity: renal failure (serum creatinine $>200 \mu \mathrm{mol} / \mathrm{l}$ ), hepatic failure, severe obstructive airway disease, severe peripheral vascular disease preventing femoral arterial access, significant aortic aneurysm, and any other co-morbidity associated with a significant increase in the risk with cardiac catheterisation.

Baseline and follow up ventriculograms were analysed off line by an automated edge detection technique. The regional left ventricular function was assessed by two methods: firstly, regional wall motion by a five segment model (anterobasal, anterolateral, apical, diaphragmatic, and posterobasal), ${ }^{10}$ and secondly, by the Slager wall motion analysis of a 20 segment model (segments 1-10 from left anterior descending coronary artery territory and segments 11-20 from left circumflex/right coronary artery territory). ${ }^{11}$

\section{Coronary fractional flow reserve and pre/post PDCF} Coronary fractional flow reserve (FFRcor) was calculated from the ratio $\mathrm{Pd}-\mathrm{Pw} / \mathrm{Pa}-\mathrm{Pw}$. Pre- and post-PCI PDCF were calculated from the constancy of the equation $\mathrm{PDCF}=\mathrm{PW}-\mathrm{Pv} / \mathrm{Pa}-\mathrm{Pv}$. In this study the mean Pa during different stages of PCI was recorded. As previously mentioned Pv was not routinely recorded. However, patients who were haemodynamically unstable and likely to have increased Pv (cardiogenic shock, right ventricular infarction) were excluded and hence $\mathrm{Pv}$ was not included in the calculations $(\mathrm{Pv}=0)$.

\section{TMP and Rentrops angiographic collateral grading}

TMP grades were assessed as follows: grade 0, no blush; grade 1, minimal blush; grade 2, good blush with slow clearing of myocardial contrast; and grade 3, good blush with normal clearing of myocardial contrast.

Rentrops collateral grades were assessed as follows: grade 0 , no filling of infarct related artery with contralateral injection; grade 1, filling of small side branches of infarct related artery; grade 2, good filling of major side branches of the infarct related artery; and grade 3, excellent filling of the infarct related artery up to the segment of obstruction.

Two physicians, one of whom was blinded to the study protocol, assessed pre- and post-PCI TIMI flow, post-PCI TMP grade, and Rentrops collateral grade. In case of discrepancy (which occurred with respect to two patients) the opinion of a third physician was considered final.

\section{Statistical methods}

Analyses explored the relations between PDCF, microvascular perfusion (TMP grade), angiographic collateral grade (Rentrops), and ventricular function (LVEDP, wall motion index, and Slager wall motion index).

Mean and standard deviations were calculated for pre- and post-FFRmyo, pre- and post-FFRcor, and PDCF before PCI, during balloon inflation, and after PCI. Changes in PDCF during balloon dilatation and after PCI were compared with pre-PCI values and assessed for significant departure from zero by paired $t$ tests. Mean PDCF and Pw values for patients with differing TMP $(0$ versus $>0)$ and Rentrops collateral grades ( 0 versus $>0$ ) were compared with two sample $t$ tests (assuming unequal variance) and Pearson correlation coefficients were calculated. A probability value of $\mathrm{p}<0.05$ was considered significant, and no adjustment has been made for multiple comparisons.

To explore the relation between LVEDP and PDCF, a scatter plot was formed. Then the patients were categorised into two groups: significantly increased LVEDP ( $>20 \mathrm{~mm} \mathrm{Hg})$; and normal or mildly increased LVEDP $(<20 \mathrm{~mm} \mathrm{Hg})$. Mean PDCF was compared between these two groups by a two sample $t$ test.

Normal theory regression analyses were performed to explore the linear relations between PDCF as the explanatory variable and regional left ventricular function (as measured by the change over baseline in the regional and Slager wall motion indices). This was adjusted for the territory involved (either left anterior descending coronary artery or left circumflex/right coronary artery territories).

\section{RESULTS}

Pre-PCI FFRmyo and PDCF during initial balloon dilatation were documented in all patients. We were unable to record the post-PCI FFRmyo in four patients because of device failure. Table 1 shows demographic and patient details.

Table 2 shows the numbers of patients with each TIMI flow grade before PCI and the change in grade after PCI. Table 3 details post-PCI TMP grades of all patients.

Table 4 shows mean (SD) FFRmyo, FFRcor, and PDCF before PCI, during balloon occlusion, and after PCI $(\mathrm{n}=38)$.

\begin{tabular}{ll} 
Table 1 Details of 38 patients undergoing rescue \\
percutaneous coronary intervention (PCI) \\
\hline Age (years) mean (SD) & $56(12)$ \\
Men & $33(87 \%)$ \\
Site of acute myocardial infarction & $19(50 \%)$ \\
$\quad$ Anterior & $19(50 \%)$ \\
$\quad$ Inferior & $19(50 \%)$ \\
Thrombolytic agent used & $19(50 \%)$ \\
$\quad$ Tissue plasminogen activator & 146 \\
Streptokinase & 413 \\
Median pain to needle time (minutes) & $6(16 \%)$ \\
Median pain to PCI time (minutes) & $6(16 \%)$ \\
Diabetes & $32(84 \%)$ \\
Hypertension & $26(68 \%)$ \\
Hyperlipidaemia & $35(92 \%)$ \\
Current smoker & $29(76 \%)$ \\
Procedural details & \\
$\quad$ Stent use & \\
Glycoprotein llb/llla use & \\
\hline *Time from pain to completion of PCI. &
\end{tabular}


Table 2 Numbers of patients with each TIMI flow grade before and after $\mathrm{PCl}$

\begin{tabular}{lcllll}
\hline & \multicolumn{5}{l}{ After PCI } \\
\cline { 3 - 6 } TIMI flow grade & Before PCI & $\mathbf{0}$ & $\mathbf{1}$ & $\mathbf{2}$ & \multicolumn{1}{l}{$\mathbf{3}$} \\
\hline 0 & 19 & 0 & 0 & 3 & 16 \\
1 & 2 & 0 & 0 & 1 & 1 \\
2 & 13 & 0 & 0 & 1 & 12 \\
3 & 4 & 0 & 0 & 0 & 4 \\
\hline
\end{tabular}

\section{PDCF and TMP grade}

Mean (SD) PDCF in patients with TMP grade 0 after PCI was $0.30(0.11)$ and in patients with TMP $1-3$ grades was 0.15 (0.07) (fig 1) (two sample $t$ test $\mathrm{p}<0.0001$, Pearson correlation $r=-0.5)$. A similar relation was observed between Pw and TMP grade (mean (SD) 28 (16) with TMP $0 \vee 9$ (7) with TMP $>0, \mathrm{p}=0.001, r=-0.4)$.

\section{PDCF and LVEDP}

LVEDP was $>20 \mathrm{~mm} \mathrm{Hg}$ in 21 patients and $<20 \mathrm{~mm} \mathrm{Hg}$ in 17 patients. An increased LVEDP was associated with higher PDCF index, which almost reached significance $(0.28(0.14)$ with LVEDP $>20 \mathrm{~mm} \mathrm{Hg} \quad v \quad 0.22$ (0.09) with LVEDP $<20 \mathrm{~mm} \mathrm{Hg}, \mathrm{p}=0.08, r=0.2$ ) (fig 2).

\section{PDCF and Rentrops collateral grade}

Angiographic collaterals were not evident in 26 patients (grade 0). Eleven patients had grade 1 and one patient had grade 2 collaterals. There was no statistical relation between PDCF and Rentrops collateral grade $(0.26(0.13)$ with grade 0 $v 0.25$ (0.11) with grades $>0, \mathrm{p}=0.4, \mathrm{r}=-0.06$ ) (fig 3 ).

\section{PDCF and left ventricular function}

Left ventricular function had improved in 18 patients and deteriorated in 15 patients on follow up ventriculography at six months. Mean (SD) PDCF in patients with improved LV function was $0.25(0.13) \quad v 0.26(0.12)$ in those with deteriorated function $(\mathrm{p}=0.4)$. No linear relation was found between change in regional wall motion or Slager wall motion and a 0.1 unit increase in PDCF (table 5).

\section{DISCUSSION}

Coronary collateral blood flow can be quantified with the pressure or Doppler wire. ${ }^{12}{ }^{13}$ This can be done only in the setting of PCI, as it requires the measurement of Pwabsolute, mean Pd during balloon inflation, and complete coronary occlusion. The ratio of mean $\mathrm{Pw}$ to mean Pa during balloon inflation is PDCF ( $\mathrm{PW}-\mathrm{Pv} / \mathrm{Pa}-\mathrm{Pv}$ ). Provided that mean $\mathrm{Pv}$ and mean $\mathrm{Pa}$ are known, the theoretical mean $\mathrm{Pw}$ before and after PCI can be calculated from the constancy of the above equation. This will enable the estimation of FFRcor, which is a direct measure of antegrade coronary flow. ${ }^{1-5}$ In this study mean Pv was not routinely estimated but patients who were haemodynamically unstable and likely to have increased $\mathrm{Pv}$ were excluded. Hence, $\mathrm{Pv}$ was

\begin{tabular}{lc}
$\begin{array}{l}\text { Table } 3 \text { TIMI myocardial perfusion (TMP) } \\
\text { grades after PCl }\end{array}$ \\
\hline TMP grade & Number \\
\hline 0 & $26(68 \%)$ \\
1 & $3(8 \%)$ \\
2 & $8(21 \%)$ \\
3 & $1(3 \%)$ \\
\hline
\end{tabular}

Table 4 Myocardial fractional flow reserve (FFRmyo), coronary fractional flow reserve (FFRcor), and pressure derived coronary collateral flow (PDCF) index before PCl, during balloon inflation, and after $\mathrm{PCl}$

\begin{tabular}{llll}
\hline & FFRmyo & FFRcor & PDCF \\
\hline Before PCl & $0.49(0.24)$ & $0.34(0.29)$ & $0.15(0.11)$ \\
During occlusion & & & $0.25(0.12)$ \\
After PCl & $0.94(0.04)$ & $0.91(0.06)$ & $0.03(0.03)$ \\
\hline \multicolumn{2}{l}{ Data are mean (SD). } & & \\
\end{tabular}

considered to be 0 and the equation was simplified to $\mathrm{PDCF}=\mathrm{Pw} / \mathrm{Pa}$

PDCF has been shown in prospective studies to predict future ischaemic events after PCI in patients with stable angina and normal left ventricular function. A Pw $-\mathrm{Pv} /$ $\mathrm{Pa}-\mathrm{Pv}$ of $<0.28$ or $\mathrm{Pw} / \mathrm{Pa}$ of $<0.30$ was associated with a nearly five times greater risk of a future ischaemic event, which was evident even at five years after the intervention. ${ }^{6}$

Studies in the setting of acute myocardial infarction are sparse. A PDCF index of $>24 \%$ (that is, PDCF $>0.24$ ) has been shown to be the strongest predictor of wall motion recovery after primary PCI. This was independent of time to reperfusion. This study selected patients who achieved complete reperfusion angiographically (TIMI 3 flow) after primary PCI. Blush scoring was not done to subselect patients with microcirculatory dysfunction, and those with lower TIMI flows were excluded. ${ }^{7}$ One of the problems with the use of the pressure wire in the setting of acute myocardial infarction is microvascular dysfunction and loss of viable myocardium, which can theoretically reduce the maximum hyperaemic response to both intravenous and intracoronary stimuli. This is highlighted by the results obtained by Yamamoto and colleagues $^{8}$ in the same clinical setting. In their study patients with lower grades of TIMI flow after PCI were not excluded and microvascular function was assessed by contrast echocardiography at the end of the procedure. Two groups were identified: those with contrast echocardiography no reflow and those with contrast echocardiography reflow. PDCF was significantly higher in the contrast echocardiography no reflow group and, moreover, this correlated inversely with the extent of regional wall motion improvement at 28 days. The authors

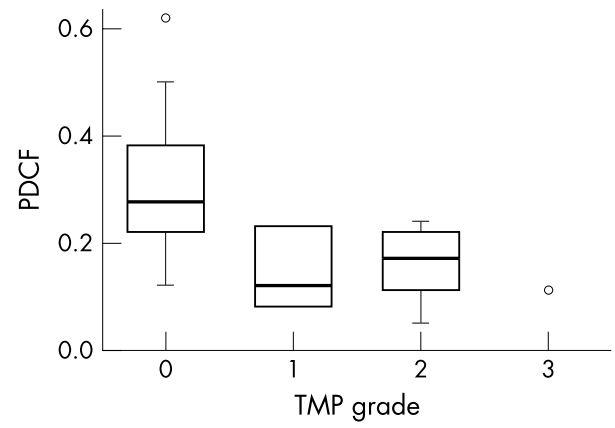

Figure 1 Box plot of pressure derived coronary collateral flow (PDCF) index and tissue myocardial perfusion (TMP) grade. The lower, middle, and upper lines of the box represent the 25th, 50th, and 75th centiles, respectively. The lower and upper tails are the lower and upper adjacent points, which reflect the spread of the data. The relation between PDCF and change in TMP group is expressed as a coefficient of variation $(-0.071,95 \%$ confidence interval $(\mathrm{Cl})-0.109$ to -0.033 ; $p<0.0011$. The lower adjacent point is the smallest data point $\geqslant x(25)-1.5 x$ (interquartile) and the upper adjacent point is largest data point $\leqslant x(75)+1.5 x$ (interquartile). Outside values (circles) are any points more extreme than the upper and lower adjacent values. 


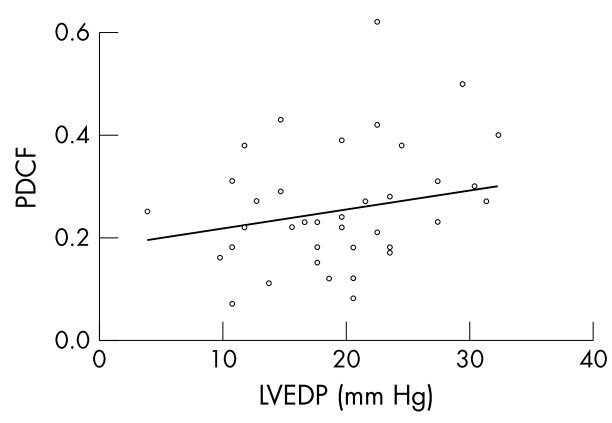

Figure 2 Relation between PDCF and left ventricular end diastolic pressure (LVEDP). The regression equation for the relation between PDCF and LVEDP is PDCF $=0.180+0.00364($ LVEDP $) ; p=0.2$.

concluded that PDCF in this clinical setting more closely reflects microcirculatory dysfunction than collateral flow. ${ }^{8}$ The increased Pw and thereby higher PDCF may also be affected by reduced myocardial contractility or increased LVEDP. More recently, PDCF in patients with TIMI 3 flow after thrombolysis (PCI performed at a mean of five days after an acute myocardial infarction) was inversely correlated with resolution of ST segments. ${ }^{9}$

Similar studies in the setting of rescue PCI are of interest because microcirculatory dysfunction is the rule rather than the exception. When failed reperfusion is diagnosed by standard ECG criteria the usual finding at angiography is thrombotic occlusion of the infarct related artery. However, ECG evidence of failed reperfusion may persist despite an open artery with TIMI 2 or 3 flow. These patients have poor or absent tissue perfusion and wall motion abnormalities that deteriorate on pharmacological stress testing. It is important to recognise this group of patients, as their outcomes are poor compared with those of patients who achieve complete reperfusion. ${ }^{14}$

TMP grading has emerged as a semiquantitative angiographic method of assessment of myocardial microcirculatory function after reperfusion either by thrombolysis or by PCI. In patients with TIMI 3 flow after thrombolysis, normal or near normal tissue reperfusion (TMP 2/3) is associated with superior clinical outcomes compared with abnormal tissue reperfusion (TMP 0/1). ${ }^{15}$ Stone and colleagues ${ }^{16}$ have shown a similar relation in the setting of primary and rescue PCI. In

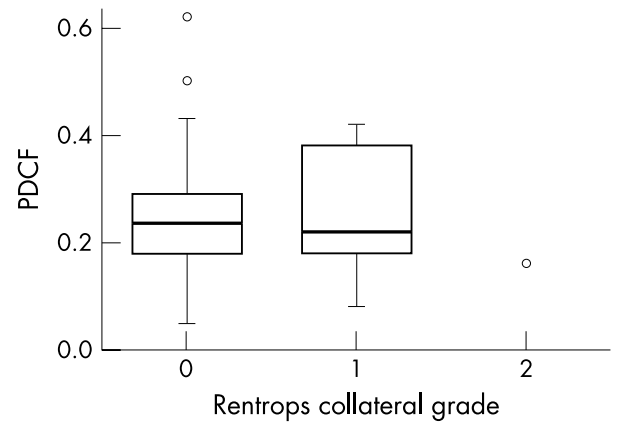

Figure 3 Box plot of PDCF and Rentrops collateral grade. The lower, middle, and upper lines of the box represent the 25th, 50th, and 75th centiles, respectively. The lower and upper tails are the lower and upper adjacent points, which reflect the spread of the data. There was no relation between PDCF and Rentrops collateral flow (coefficient of variation $-0.0143,95 \%$ confidence interval -0.0916 to 0.0629 , $\mathrm{p}=0.7)$. The lower adjacent point is the smallest data point $\geqslant x(25)-1.5 x$ (interquartile) and the upper adjacent point is largest data point $\leqslant x(75)+1.5 x$ (interquartile). Outside values (circles) are any points more extreme than the upper and lower adjacent values.
Table 5 Relation from a linear regression model between change over baseline in regional left ventricular function (the response, or $y$, variable, assessed by five segment and 20 segment models) and a 0.1 unit change in PDCF (the explanatory, or $x$, variable)

\begin{tabular}{|c|c|c|}
\hline Regional wall motion & $\begin{array}{l}\text { Change in regional wall motion } \\
(95 \% \text { Cl) for a } 0.1 \text { unit increase } \\
\text { in PDCF }\end{array}$ & p Value \\
\hline $\begin{array}{l}\mathrm{AB}+\mathrm{AL}+\mathrm{AP} \\
\mathrm{DM}+\mathrm{PB}+\mathrm{AP} \\
\text { Slager segments } 1-10 \\
\text { Slager segments } 11-20\end{array}$ & $\begin{array}{r}3.2(-3.2 \text { to } 9.6) \\
-0.2(-8.0 \text { to } 8.4) \\
-0.8(-3.4 \text { to } 1.8) \\
-2.1(-1.4 \text { to } 5.6)\end{array}$ & $\begin{array}{l}0.32 \\
0.97 \\
0.53 \\
0.24\end{array}$ \\
\hline
\end{tabular}

Anterobasal ( $A B)$, anterolateral (AL), and apical (AP) segments are from the left anterior descending coronary artery (LAD) territory in the five segment model; diaphragmatic (DM), posterobasal (PB), and AP segments are from the left circumflex (LCX)/right coronary artery (RCA) territories. Note: the AP segment overlaps the two territories. Slager segments $1-10$ represent LAD territory and segments 11-20 represent $\mathrm{LCX} / \mathrm{RCA}$ territory.

$\mathrm{Cl}$, confidence interval.

163 of the 171 patients who achieved TIMI 3 flow after PCI, one year cumulative mortality was strongly correlated with TMP grade after PCI (6.8\% with TMP grade $3 v 13 \%$ with TMP grade $2 \vee 22 \%$ with TMP grade $0 / 1, p=0.002)) .{ }^{16}$ Infarct sizes are smaller in patients who achieve superior TMP grade after reperfusion. ${ }^{17}$ Subsequent ventricular remodelling and changes in the left ventricular function appear to be positively influenced in these patients. Despite being considered a relatively crude method of assessment of microvascular function, TMP grading has been well validated and is the simplest method available. ${ }^{15-18}$ We found a strong association between increased PDCF index and lower TMP grade (fig 1). Mean Pw was also similarly related to TMP grade.

In addition to more prolonged time to reperfusion, persistent microcirculatory dysfunction despite technically successful PCI is likely to be responsible for inferior outcomes with rescue PCI compared with successful thrombolysis and primary PCI. In this study, 26 patients $(68 \%)$ had TMP grade 0 after rescue PCI despite TIMI 3 flow in the epicardial artery. Downstream microvascular obstruction, $\alpha$ adrenergic neural reflexes, spasm, or thrombotic occlusions of microvessels contribute to the impaired microvascular perfusion in these patients. ${ }^{19}$ Additional treatment with glycoprotein IIb/IIIa blockers or intracoronary vasodilators (nitrates, verapamil, adenosine) may improve the microcirculation, but evidence of improved outcomes is lacking except in the case of abciximab. ${ }^{20}$

LVEDP in acute myocardial infarction is strongly influenced by the status of the systolic function and increased LVEDP is a marker of infarct size. ${ }^{2122}$ Patients with significantly increased LVEDP (> $20 \mathrm{~mm} \mathrm{Hg}$ ) had increased PDCF compared with patients with normal or mildly increased LVEDP $(<20 \mathrm{~mm} \mathrm{Hg})$, which nearly achieved significance (fig 2). Furthermore, we found no correlation between PDCF and Rentrops angiographic collateral grade (fig 3).

We found no linear relation between changes in regional left ventricular function and PDCF. This suggests that PDCF in the clinical setting of this study cannot predict outcomes in left ventricular function. This is further emphasised by the similar mean PDCF values and the wide overlap of this index between patients with improved and patients with deteriorated left ventricular function.

\section{Limitations}

The number of patients in this study was relatively small, which will have reduced our ability to detect reliably what 
may be clinically important relations between the study measurements.

It is possible that the inability of PDCF to predict outcomes in left ventricular function was influenced by this limited number of patients, with the wide confidence intervals around the estimated effects not ruling out some possibly large effects. However, the wide overlap of PDCF values between patients with and without improved regional function suggests an absence of such an association.

Small changes may occur in Pv during PCI and this may affect the calculated $\mathrm{Pw}$ and hence the pressure wire derived collateral flow. These changes did not affect measured PDCF and TMP conclusions in this study.

Intravenous rather than intracoronary adenosine does result in a more sustained period of maximum hyperaemia. However, provided that doses of intracoronary adenosine are adequate-as in this study-maximum hyperaemia comparable with intravenous administration is attained but for a shorter duration. ${ }^{23}$ Moreover, we have previously performed these measurements with intravenous adenosine and found no impact on the calculated PDCF.

TMP grading is a semiquantitative method for assessment of microvascular function and has its limitations. It may be less accurate than other techniques, such as contrast echocardiography, dobutamine stress echocardiography, and cardiac magnetic resonance imaging. ${ }^{24-26}$ However, the simplicity of TMP grading makes it an attractive technique and its clinical utility has been established in several studies. ${ }^{15-17} 27$ We used the method described by Gibson and colleagues. ${ }^{15}$ It is possible that slightly different results may have been obtained if the alternative method described by van't Hof and colleagues had been used. ${ }^{18}$ However, both methods have been validated and adopted.

\section{Conclusion}

In the clinical setting of failed reperfusion after thrombolysis for acute myocardial infarction, the PDCF index does not predict outcomes in left ventricular function. Higher PDCF is associated with lower TMP grade, increased LVEDP, and angiographic evidence of microvascular dysfunction and does not truly reflect collateral protection. We postulate that the PDCF index in acute myocardial infarction is invalid and that $\mathrm{Pw}$ reflects a dysfunctional microcirculation.

\section{ACKNOWLEDGEMENTS}

Part of this study was presented as an oral contribution in the Young Investigator's Award session at the ESC Scientific Congress, Berlin, Germany and was published in abstract form in European Heart Journal $(2002 ; 23: 488)$.

\section{Authors' affiliations}

K P Balachandran, Bristol Royal Infirmary, Bristol, UK

C Berry, Glasgow Royal Infirmary, Glasgow, UK

J Norrie, Centre for Healthcare Randomised Trials, Health Services

Research Unit, University of Aberdeen, Aberdeen, UK

B D Vallance, M Malekianpour, T J Gilbert, A C H Pell, Lanarkshire

Acute Hospitals NHS Trust, East Kilbride, UK

K G Oldroyd, Western Infirmary, Glasgow, UK

Conflict of interest and financial disclosures: none.

Site of study: Lanarkshire Cardiac Catheterisation Laboratories, Hairmyres Hospital, Lanarkshire Acute Hospitals NHS Trust, East Kilbride

\section{REFERENCES}

1 Pijls NHJ, De Bruyne B. The fractional flow reserve. In: Pijls NHJ, De Bruyne B, eds. Coronary pressure. Dordrecht: Kluwer Academic Publishers, 2000:51-83.
2 Piils NHJ, De Bruyne B, Peels K, et al. Measurement of fractional flow reserve to assess the functional recovery of coronary artery stenosis. N Engl J Med 1996;334:1703-8.

3 Kern MJ, De Bruyne B, Pijls NHJ. From research to clinical practice: current role of intracoronary physiologically based decision making in the cardiac catheterisation laboratory. J Am Coll Cardiol 1997;30:613-20.

4 Pijls NHJ, De Bruyne B. Coronary pressure measurement and fractional flow reserve. Heart 1998;80:539-42.

5 Piils NHJ, Van Son JMA, Kirkeeide RL, et al. Experimental basis of determining maximum coronary, myocardial and collateral blood flow by pressure measurements for assessing functional stenosis severity before and after percutaneous transluminal coronary angioplasty. Circulation 1993;87:1354-67.

6 Pijls NH, Beck GJ, El Gamal MI, et al. Quantification of recruitable coronary collateral blood flow in conscious humans and its potential to predict future ischaemic events. J Am Coll Cardiol 1995;25:1522-8.

7 Lee CW, Park SW, Cho GY, et al. Pressure derived fractional collateral blood flow: a primary determinant of left ventricular recovery after reperfused acute myocardial infarction. J Am Coll Cardiol 2000;35:949-55.

8 Yamamoto $\mathrm{K}$, Ito $\mathrm{H}$, Iwakura $\mathrm{K}$, et al. Pressure derived collateral flow index as a parameter of microcirculatory dysfunction in acute myocardial infarction. $J$ Am Coll Cardiol 2001;38:1383-9.

9 Sezer M, Nisanci Y, Umman B, et al. New support for clarifying the relation between ST segment resolution and microvascular function: degree of ST segment resolution correlates with the pressure derived collateral flow index. Heart 2004;90:146-50

10 Wong WH, Kirkeeide RL, Gould KL. Computer applications in angiography. In: Collins SM, Skorton DJ, eds. Cardiac imaging and image processing. New York: McGraw-Hill, 1986.

11 Slager CJ, Hooghoudt THE, Serruys PW, et al. Quantitative assessment of regional left ventricular motion using endocardial landmarks. J Am Coll Cardiol 1986:7:317-26.

12 Seiler C, Fleisch M, Garachemani A, et al. Coronary collateral quantitation in patients with coronary artery disease using intravascular flow velocity or pressure measurements. J Am Coll Cardiol 1998;32:1272-9.

13 Billinger M, Kloos P, Eberli $F$, et al. Physiologically assessed coronary collateral flow and adverse cardiac ischaemic events: a follow-up study in 403 patients with coronary artery disease. J Am Coll Cardiol 2002;40:1545-50.

14 Ito H, Tomooka T, Sakai N, et al. Lack of myocardial perfusion immediately after successful thrombolysis: a predictor of poor recovery of left ventricular function in anterior myocardial infarction. Circulation 1992:85:1699-705.

15 Gibson CM, Cannon CP, Murphy SA, et al. Relationship of TIMI myocardial perfusion grade to mortality after administration of thrombolytic drugs. Circulation 2000;101:125-30

16 Stone GW, Peterson MA, Lansky AJ, et al. Impact of normalised myocardial perfusion after successful angioplasty in acute myocardial infarction. J Am Coll Cardiol 2002;39:591-7.

17 Angeja BG, Gunda M, Murphy SA, et al. TIMI myocardial perfusion grade and ST segment resolution: association with infarct size as assessed by single photon emission computed tomography imaging. Circulation 2002; 105:282-5

18 van't Hof AW, Liem A, Suryaprananta H, et al. Angiographic assessment of myocardial perfusion in patients treated with primary angioplasty for acute myocardial infarction: myocardial blush grade. Zwolle myocardial infarction study group. Circulation 1998:97:2302-6.

19 Gregorini L, Marco J, Kozakova M, et al. Alpha adrenergic blockade improves recovery of myocardial perfusion and function after coronary stenting in patients with acute myocardial infarction. Circulation 1999:99:482-90.

20 Lefkovits J, Ivanhoe RJ, Califf RM, et al. Effects of platelet glycoprotein Ilb/Illa receptor blockade by a chimeric monoclonal antibody (abciximab) on acute and six month outcomes after percutaneous transluminal angioplasty for acute myocardial infarction. EPIC investigators. Am J Cardiol 1996;77:1045-51.

21 Pirzada FA, Ekong EA, Vokonas PS, et al. Experimental myocardial infarction. Sequential changes in left ventricular pressure-length relationship in the acute phase. Circulation 1976;53:970-5.

22 Shell W, Peter T, Mickle D, et al. Prognostic implication of reduction of left ventricular filling pressure in early transmural acute myocardial infarction. Am Heart J 1981; 102:335-40.

23 De Bruyne B, Pijls NH, Barbato A, et al. Intracoronary and intravenous adenosine $5^{\prime}$-triphosphate, adenosine, papaverine, and contrast medium to assess fractional flow reserve in humans. Circulation 2003;107:1877-83.

24 Czitrom D, Karila-Cohen D, Brochet $E$, et al. Acute assessment of microvascular perfusion patterns by myocardial contrast echocardiography during myocardial infarction: relation to timing and extent of functional recovery. Heart 1999:81:12-6.

25 Hillis GS, Mulvagh SL, Pellikka PA, et al. Comparison of intravenous myocardial contrast echocardiography and low-dose dobutamine stress echocardiography for predicting left ventricular recovery following acute myocardial infarction. Am J Cardiol 2003;92:504-8.

26 Beek AM, Kuhl HP, Bondarenko O, et al. Delayed contrast-enhanced magnetic resonance imaging for the prediction of regional functional improvement after acute myocardial infarction. J Am Coll Cardiol 2003;42:895-901.

27 Haager $P$, Christott $P$, Heussen $N$, et al. Prediction of clinical outcome after mechanical revascularisation in acute myocardial infarction by markers of myocardial reperfusion. J Am Coll Cardiol 2003;41:532-8. 